\title{
Multifractal analysis of interplanetary magnetic field obtained during CME events
}

\author{
M. J. A. Bolzan ${ }^{1}$ and R. R. Rosa ${ }^{2}$ \\ ${ }^{1}$ Laboratório de Física, Universidade Federal de Goiás, Campus de Jataí, UFG, Brazil \\ ${ }^{2}$ Laboratório Associado de Computação e Matemática Aplicada, Instituto Nacional de Pesquisas Espaciais, INPE, Brazil \\ Correspondence to: M. J. A. Bolzan (mauricio.bolzam@pq.cnpq.br)
}

Received: 1 March 2012 - Revised: 3 July 2012 - Accepted: 6 July 2012 - Published: 1 August 2012

\begin{abstract}
In this paper, we have analyzed the interplanetary magnetic field (IMF) obtained by ACE satellite during the coronal mass ejection events. The characterization of the IMF fluctuations was performed using the singular power spectra deviations obtained from a wavelet transform modulus maxima (WTMM) approach. The results suggest the existence of different multifractal processes driving the intermittency during and after the CME events on the IMF time series. The characteristic time scales found, using the WTMM, and possible related physical mechanisms are discussed in the context of nonlinear interplanetary magnetic field response.
\end{abstract}

Keywords. Interplanetary physics (Interplanetary magnetic fields)

\section{Introduction}

Geophysics time series are often generated by complex spatio-temporal dynamics of which nonlinearity and scaling are the most important processes. In particular, the time series from a space environment such as the interplanetary magnetic field (IMF) presents several kinds of phenomena which are important to study due to the consequences over the Earth (Gonzalez et al., 1999). It presents a regular component (e.g. the solar daily variation or "Sq"), whereas it presents other irregular components due to geomagnetic disturbances caused by the solar wind-magnetosphere coupling, which is a remarkable nonlinear process. From the point of view of space weather, the study of irregular geomagnetic oscillations in solar-terrestrial physics is very important due to adverse effects on data transmission by satellites, power lines, and many others. Thus, the systematic study of the statistical properties of complex magnetospheric variability and their relations to the solar wind has attracted a growing interest (e.g. Chapman et al., 1998; Chang, 1999; Kovács et al., 2001; Lui, 2002; Vörös et al., 2002). Recently, Papa et al. (2006) and Papa and Sosman (2008), who have been working with time series of the geomagnetic field time series obtained during disturbances, have shown that it is possible to find some universal characteristics such as a threshold system as the source of such perturbations.

The main issue in the analysis and interpretation of the IMF time series is to describe the multifractal aspects of the CME events. The intermittency leads to deviation from usual Kolmogorov turbulent velocity structure functions (Kolmogorov, 1941), and its main signature is the spectrum of singularity exponents, $f(\alpha)$, which represent a power-law scaling-free dependence (Frisch, 1995). For solar wind turbulence, the so-called multifractal p-model (Halsey et al., 1986; Meneveau and Sreenivasan, 1987) describes how solar wind energy can be distributed among scales following a multiplicative rescaling structure. Actually, the multifractal analysis, in contrast to the traditional power spectrum analysis, has shown that the Holder exponents, for local singularity, are time-dependent showing that the flux energy at a given scale is not homogeneously distributed in time as in the traditional homogenous $1 / f^{\alpha}$ turbulent spectrum. This fact suggests that the fluctuations can be described with a multifractal scaling law associated with intermittency where other nonlinear and coherent processes can coexist (Vörös et al., 2002; Weygand et al., 2005; Wanliss et al., 2005). In our approach, it is considered that the intermittency behavior is associated with the multifractal turbulence model (Muzy et al., 1991). Thus, this study deals with the multifractal analyses of the IMF records that are fully intermittent, and, in this 

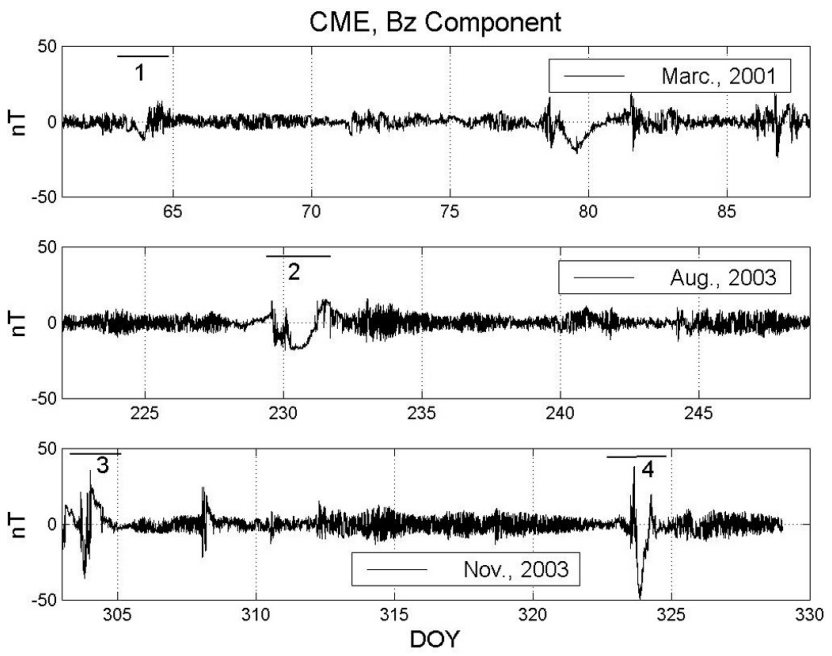

Fig. 1. Three time series of interplanetary magnetic field for months shown in legends. The CME events are indicated by a number and a short bar.

sense, the continuous wavelet transform and its maxima can be used to reveal the structure of this irregular time series and its correspondent relevant time scales.

\section{Data and methodology}

Figure 1 shows the three IMF fluctuations corresponding to the 16-s time resolution $B_{\mathrm{Z}}$-component time series observed during (a) the month of March 2001, (b) the month of August 2003, and (c) the month of November 2003 at ACE satellite (http://www.srl.caltech.edu/ACE/ASC/DATA/ level3/index.html). In this figure, regions where the CME occurred were marked according to the classification from Richardson and Cane (2004) (http://www.ssg.sr.unh.edu/ mag/ace/ACElists/ICMEtable.html), totalizing four events. Thus, the analysis has been carried out in two parts of the time series: the first part includes the CME event and the other part after the CME event. Table 1 shows the days of the year for each event. It is important to mention that the main objective was to select different events in the sense that each one has a peculiar variability pattern. Thus, we believe that we would be getting the most general results. There may be other types of events between March 2001 and November 2003. However, considering a common physical process, its statistical characteristics related to the pattern of variability should not vary significantly. Moreover, we consider the present analysis a complementary one of those events with which we had earlier work (Bolzan et al., 2005a, 2009b; Sahai et al., 2005).

Several authors have analyzed the interplanetary data records of solar wind speed (slow/fast) to study the intermittency degree (for an extensive review, see Bruno et al., 2001). Burlaga (1991) concluded that the solar wind is made
Table 1. Start and end of CME events used in this work. This classification was carried out by Richardson and Cane (2004).

\begin{tabular}{cll}
\hline Event & Start of CME & End of CME \\
\hline 1 & 4 March 2001 & 5 March 2001 \\
2 & 17 August 2003 & 20 August 2003 \\
3 & 31 October 2003 & 2 November 2003 \\
4 & 20 November 2003 & 21 November 2003 \\
\hline
\end{tabular}
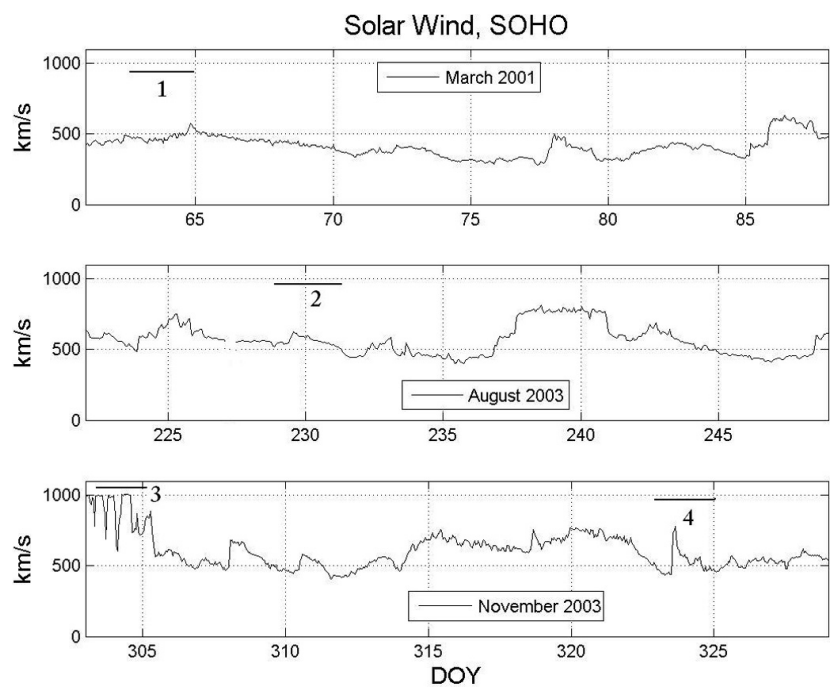

Fig. 2. Three time series of solar wind velocity for months shown in legends. The CME events also are indicated by a number and a short bar.

of a mixture of sheets and space-filling eddies of different sizes. Marsch and Liu (1993) showed that the slow solar wind is more intermittent than the fast solar wind. Bruno et al. (2001), by using a new method based on the wavelets, identified the flux tubes convected by the wind, which tangle up in space. Due to the importance of the solar wind on the intermittence in interplanetary environment, Fig. 2 shows the solar wind time series for the same periods of the IMF time series used in this work. Data sets were obtained from SOHO satellite on the home page (http://sohowww.nascom. nasa.gov/). We can notice that the first two events of CME (numbers 1 and 2) are characterized by a slow solar wind $\left(\approx 550 \mathrm{~km} \mathrm{~s}^{-1}\right)$, approximately, while the two last events are characterized by a fast solar wind (more than $750 \mathrm{~km} \mathrm{~s}^{-1}$ ). Besides, Marsch and Liu (1993) have mentioned that the slow solar wind is more intermittent than the fast solar wind; both kinds of solar winds contribute to intermittence on interplanetary environment.

Recent studies on scaling and singularity characteristics of magnetospheric fluctuations into the MHD flow system, considering solar wind and ionospheric phenomena, suggest that the near-Earth magnetosphere is a non-homogenous interconnecting multi-scale environment where spatio-temporal 
variability is intermittent and multifractal turbulence can coexist with coherent global processes and self-organized criticality (Vörös et al., 2002). As the inhomogeneity involves the singular behavior of the energy distribution in physical space resulting in strong gradients, or intermittency in the timeseries of the energy related physical quantities of the system, e.g. velocity (Rodrigues Neto et al., 2001; Ramos et al., 2004), temperature (Bolzan et al., 2002) or magnetic fields (Kovács et al., 2001; Lui, 2002), an appropriate characterization of the IMF fluctuations is insured based on the multifractal spectra, $\tau(q)$, and singularity spectra, $f(\alpha)$, which quantifies the deviations of the observed singularities $\alpha$ from the expected value and gives a measurement of the intermittency level (Vörös, 2000). The underlying time scales involved in the intermittent level and their different types of $\tau(q)$ and $f(\alpha)$ spectra can characterize the fluctuation-driven mechanism, for example, involving coherent structures or intermittent interplanetary magnetic field responses from CME events influence.

Among a few methods for multifractal analysis, we have considered, in order to obtain the singularity spectrum, $f(\alpha)$, the wavelet transform modulus maxima (WTMM) (Muzy et al., 1991). The basic idea behind the WTMM method is to describe the partition function over only the modulus maxima of the wavelet transform of the signal. Thus, as given by Enescu et al. (2006), this transform is written as

$$
(W f)(s, b)=\frac{1}{s} \int f(t) \psi^{*}\left[\frac{t-b}{s}\right] d t,
$$

where $s$ is the scaling factor, $b$ is the location parameter, $\psi^{*}$ is the complex conjugate of the continuous wavelet function and $f(t)$ is the time series under analysis. Due to the variability pattern of our data, we have used the Morlet wavelet function given by

$$
\psi(t)=e^{i K t} \cdot e^{-t^{2} / 2},
$$

where $K=5$ is recommended in practice, but can be modified. The scaling and translation of this wavelet function are performed by the parameters $s$ and $b$. While the scale parameter $s$ stretches (or compresses) the mother wavelet to the required resolution, the translation parameter $b$ shifts the basis functions to the desired location.

It can be shown that the wavelet transform can reveal the local characteristics of $f$ at a point $x_{0}$. More precisely, we have the following power-law relation:

$$
W f\left(s, x_{0}\right) \approx|s|^{\alpha\left(x_{0}\right)},
$$

where $\alpha\left(x_{0}\right)$ is the Hölder exponent (or singularity strength).

The wavelet transform of $f(t)$ is able to show the invariance with respect to some renormalization operations involving multiplicative cascades. This means that there is a hierarchy of the WTMM that has been used to define the partition function based on the multifractal formalism (Arneodo et al., 1995). The moment $q$ of the measure distributed on the WTMM hierarchy is used to define the dependence of the scaling function on the moment $q$, so that the partition function $Z(q, s)$, of the $q$-th moment of the measure distributed over the WTMM at the scale $s$, and it can be written as

$Z(q, s)=\sum_{n}\left|W f\left(y_{n}, s\right)\right|^{q}$,

where $y_{n}$ is the position of all local maxima at a fixed scale $s$. Often scaling behavior observed for $Z(q, s)$ and the spectrum $\tau(q)$, which describes how $Z$ scales with $S$, can be defined as follows:

$Z(q, s) \approx S^{\tau(q)}$.

Then, the multifractal spectrum is obtained by applying the Legendre transformations to $\tau(q)$. If there is a system that has a monofractal structure, then we obtain a straight line in linear plot of $\tau$ versus $q$. When the $\tau(q)$ exponents in Eq. (5) are in the form of straight line, the time series is a monofractal; otherwise, the time series is a multifractal (Oswiecimka et al., 2006).

\section{Results and discussion}

In order to analyze the influence of quasi-periodic oscillations from CME on the multifractal spectra, we applied the Eq. (5) in the four time series with CME part and after CME time series ones. The multifractal spectra method was applied to the length of the time series equivalent to the duration of the CME intervals, as shown on Table 1. Thus, we use the same length of the time series after the same CME events.

Figure 3 shows the values of the $\tau$-spectrum for all the time-series data. Note that the both $\tau$-spectra are curved, indicating the multifractal nature. However, the $\tau$-spectrum for the case where the CMEs are present is less curved, if compared with results after these CME events. Thus, it is possible to conjecture that, during the CME events, there are physical phenomena that are well defined, such as magnetic coherent structures, as shown in Fig. 5 (Chang et al., 2006).

By visual inspection in Fig. 4, where we plotted the mean values of the singularity spectrum $f(\alpha)$ for both categories (during the CMEs and after CME events), we note that in the both curves there is a stronger multifractality degree. It is due to the large $w=\alpha_{\max }-\alpha_{\min }$, which is observed during and after CME. It is noteworthy that, although the $w$ parameters are very closed $(w=1.16$ during the CME and $w=1.19$ after the CME), the values for $\alpha_{\max }$ are slightly different: $\alpha_{\max }=1.12$ (during the CME) and $\alpha_{\max }=0.91$ (after the CME). In our previous work (Bolzan et al., 2009c), by using the $\mathrm{H}$-component of the geomagnetic field obtained in Brazil, we found greatest values of the $\alpha_{\max }$ for time series with strong presence of the deterministic phenomena, such as solar daily variation on geomagnetic field. This fact indicates that the presence of the strong and deterministic phenomena 


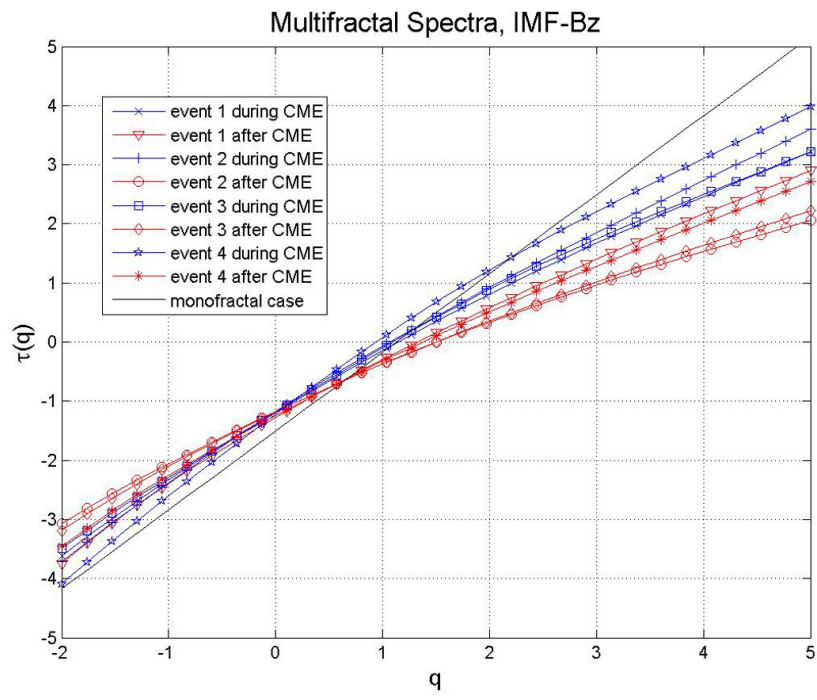

Fig. 3. Multifractal spectral from time series during CME events, after CME events and monofractal case (straight black line). The legends indicate every event studied.

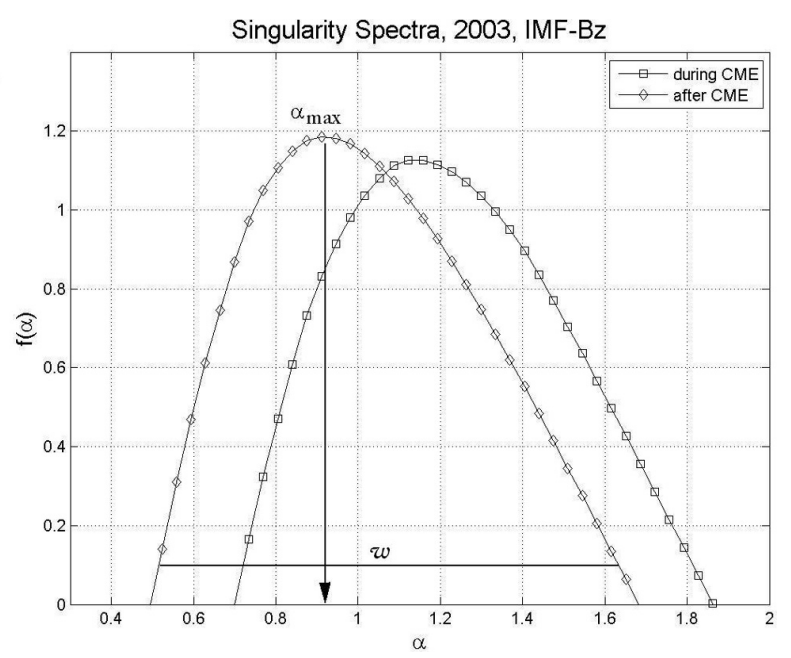

Fig. 4. Mean values of the singularity spectra, $f(\alpha)$, for two categories: with and without CME events.

contributes to decrease the multifractal characteristics on the time series. The same result also was observed in another kind of the time series, specifically the vertical total electron content (VTEC) (Bolzan et al., 2009a).

Before we try to explain both distinct behaviors (during and after CME), it is necessary to have in mind the following concepts. Chang et al. (2006) have defined the term of dynamical complexity which refers to the nonlinearly interacting dynamics among magnetic coherent structures of different sizes. One kind of such structures is shown in Fig. 5.

According to the idea mentioned before, it is possible to visualize the action of these structures over an IMF $B_{\mathrm{Z}}$ component time series of November 2003. Figure 6 shows a

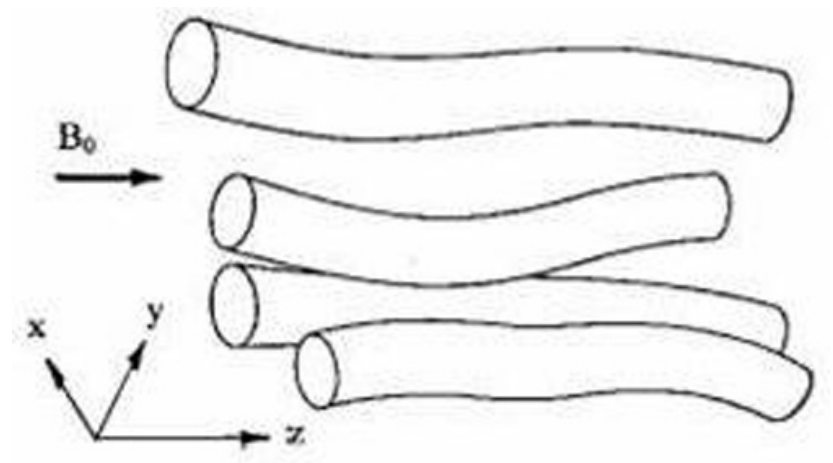

Fig. 5. Magnetic coherent vortex. Figure extracted from work of Chang et al. (2006).

schematic approach. These structures are energetic physical phenomena that have a geometric form predominant in the CME, causing an almost well-deterministic behavior that reduces the multifractality degree, because this phenomenon privileges only a few scales. After the CME, the system presents several kinds of the small structures and its different sizes, causing the increase of multifractality degree, where this elevation of the multifractal degree is necessary to promote the re-distribution of energy from CME down to dissipation scales.

Our result corroborates with results from Telesca et al. (2004), who found also a decrease of multifractality degree in geomagnetic time series due to the presence of the powerful oscillations. Thus, it is possible to conjecture that, for the CME events, there are physical phenomena that are well defined, such as magnetic coherent structures, and are responsible for the decrease of the multifractality degree observed in our results.

Following a complementary approach, non-extensive statistics has been used to understand the nonlinear magnetic processes in several space physics phenomena (e.g. Bolzan et al., 2005b; Balasis et al., 2008). Recently, this type of statistical analysis was applied to study the variability of the Dst index and also detected the presence of two characteristic dynamic regimes: (i) a variability pattern of high organization associated with intense storms, and (ii) another more irregular variability pattern associated with intermittent disturbances (Balasis et al., 2008). Although these studies have been performed for time series of the Dst index, they are associated with the same system that responds by geomagnetic activity. In this sense, these results, in conjunction with what we find here, suggest that the presence of coherent structures reduces the degree of multifractality of the system and may modulate the degree of organization of nonlinear processes in the interplanetary space. 


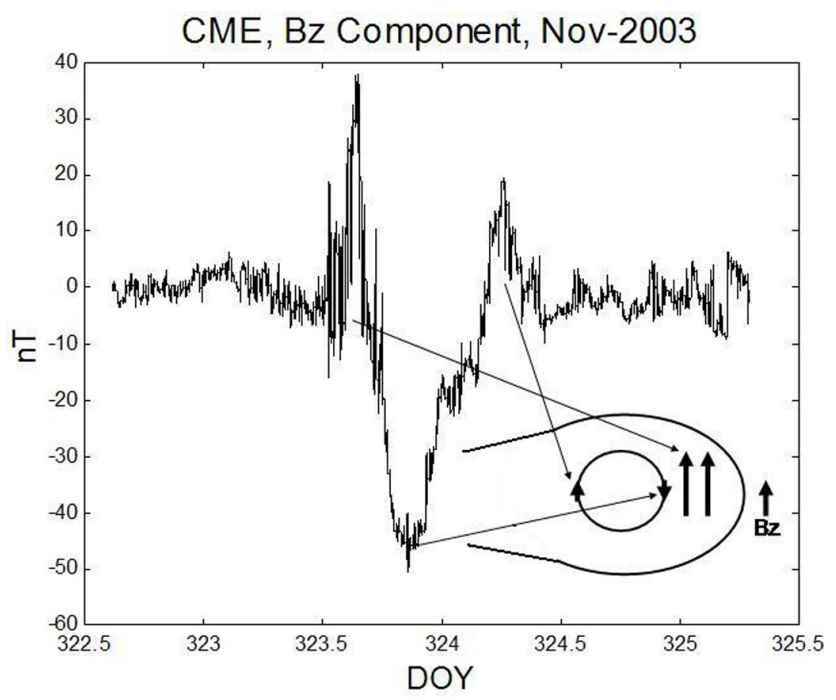

Fig. 6. Schematic approach of the action of the magnetic coherent vortex over IMF- $B_{\mathrm{Z}}$ component.

\section{Concluding remarks}

In this article, we analyze a set of characteristic data of the IMF- $B_{\mathrm{Z}}$ component obtained by ACE satellite during the coronal mass ejection (CME) events. These events are interpreted as a nonlinearly interacting dynamical system characterized by the presence of large-scale coherent structures of different sizes (Chang et al., 2006). Our multifractal analysis showed that the presence of these strong and well-behaved structures in the CME decreases the multifractality degree, if compared with periods after the CME events. This result corroborates with Telesca et al. (2004), who have also observed this behavior on geomagnetic time series where, according to them, the multifractality degree of the signal is strongly reduced by the presence of powerful oscillations. This behavior was also observed in our previous works using a different kind of time series from geomagnetic fluctuations and related data (Bolzan et al., 2009a, c). Our results, together with others which characterize the global environment interplanetary plasma, suggest that low values of the degree of multifractality can be directly associated with the presence of coherent structures. Thus, the technique used here serves as a tool to detect coherent structures present in the geomagnetic dynamics, especially when studied within the context of solar-terrestrial physics.

Acknowledgements. This work was supported by CNPq (grants no. 301457/2009-3). Thanks also to referees for fruitful comments given by this work.

Topical Editor R. Nakamura thanks two anonymous referees for their help in evaluating this paper.

\section{References}

Arneodo, A., Bacry, E., and Muzy, J. F.: The thermodynamics of fractals revisited with wavelets, Physica A, 213, 232-275, 1995.

Balasis, G., Daglis, I. A., Papadimitriou, C., Kalimeri, M., Anastasiadis, A., and Eftaxias, K.: Dynamical complexity in Dst time series using non-extensive Tsallis entropy, Geophys. Res. Lett., 35, L14102, doi:10.1029/2008GL034743, 2008.

Bolzan, M. J. A., Ramos, F. M., Sá, L. D. A., Rodrigues Neto, C., and Rosa, R. R.: Analysis of fine-scale canopy turbulence within and above na Amazon Forest using Tsallis' generalized thermostatistics, J. Geophys. Res., 107, 8063, doi:10.1029/2001JD000378, 2002.

Bolzan, M. J. A., Sahai, Y., Fagundes, P. R., Rosa, R. R., Ramos, F. M., and Abalde, J. R.: Intermittency analysis of geomagnetic storm time-series observed in Brazil, J. Atmos. Solar-Terr. Phys., 67, 1365-1372, doi:10.1016/j.jastp.2005.06.008, 2005a.

Bolzan, M. J. A., Sahai, Y., Fagundes, P. R., Rosa, R. R., Ramos, F. M., and Abalde, J. R.: Generalized thermo statistics and wavelet analysis of solar wind and proton density variability, J. Atmos. Solar-Terr. Phys., 67, 1843-1851, doi:10.1016/j.jastp.2005.01.015, 2005b.

Bolzan, M. J. A., Becker-Guedes, F., Fagundes, P. R., Sahai, Y., Pillat, V. G., and Wrasse, C. M.: Statistical analysis of the total electron content observed at $23^{\circ} \mathrm{S}$ in the Brazilian sector, Adv. Space Res., 44, 385-394, doi:10.1016/j.asr.2009.03.012, 2009a.

Bolzan, M. J. A., Guarnieri, F. L., and Vieira, P. C.: Comparisons between two wavelet functions in extracting coherent structures from solar wind time series, Brazilian J. Phys., 39, 12-17, doi:10.1590/S013-97332009000100002, 2009b.

Bolzan, M. J. A., Rosa, R. R., and Sahai, Y.: Multifractal analysis of low-latitude geomagnetic fluctuations, Ann. Geophys., 27, 569576, doi:10.5194/angeo-27-569-2009, 2009c.

Bruno, R., Carbone, V., Veltri, P., Pietropaolo, E., and Bavassano, B.. Identifying intermittency events in the solar wind, Planet. Space Sci., 49, 1201-1210, 2001.

Burlaga, L.: Intermittent turbulence in the solar wind, J. Geophys. Res., 96, 5847-5851, 1991.

Chang, T.: Self-organized criticality, multi-fractal spectra, sporadic localized reconnections and intermittent turbulence in the magnetotail, Phys. Plasmas, 6, 4137-4145, 1999.

Chang, T., Tam, S. W. Y., and Wu, C.-C.: Complexity in Space Plasmas - A Brief Review, Space Sci. Rev., 122, 281-291, doi:10.1007/s11214-006-5957-4, 2006.

Chapman, S. C., Watkins, N. W., Dendy, R. O., Helander, P., and Rowlands, G.: A simple avalanche model as an analogue for magnetospheric activity, Geophys. Res. Lett., 25, 2397-2400, 1998.

Enescu, B., Ito, K., and Struzik, Z.: Wavelet-based multiscale resolution analysis of real and simulated time-series of earthquakes, Geophys. J. Int., 164, 63-74, doi:10.1111/j.1365246X.2005.02810.x, 2006.

Frisch, U.: Turbulence, Cambridge University Press, New York, 1995.

Gonzalez, W. D., Tsurutani, B. R., and Gonzalez, A. L. C.: Interplanetary origin of geomagnetic storms, Space Sci. Rev., 88, 529-562, 1999.

Halsey, T. C., Jensen, M. H., Kadanoff, L. P., Procaccia, I., and Shraiman, B. I.: Fractal measures and singularities - The characterization of strange sets, Physical Review A, 33, 1141-1151, 
1986.

Kolmogorov, A. N.: The local structure of turbulence in an incompressible viscous flow for very high Reynolds numbers, C. R. Acad. Sci., SSSR, 30, 301-305 (see also in Proc. R. Soc. London A., 434, 9-13, 1991), 1941.

Kovács, P., Carbone, V., and Vörös, Z.: Wavelet-based filtering of intermittent events from geomagnetic time-series, Planet. Space Sci., 49, 1219-1231, 2001.

Lui, A. T. Y.: Multiscale phenomena in the near-Earth magnetosphere, J. Atmos. Solar-Terr. Phys., 64, 125-143, 2002.

Marsch, E. and Liu, S.: Structure functions and intermittency of velocity fluctuations in the inner solar wind, Ann. Geophys., 11, 227-238, 1993.

Meneveau, C. and Sreenivasan, K. R.: Simple multifractal cascade model for fully developed turbulence, Phys. Rev. Lett., 59, 14241427, 1987.

Muzy, J. F., Bacry, E., and Arneodo, A.: Wavelets and Multifractal formalism for singular signals: Application to turbulence data, Phys. Rev. Lett., 67, 3515-3518, 1991.

Oswiecimka, P., Kwapien, J., and Drozdz, S.: Wavelet versus detrended fluctuation analysis of multifractal structures, Phys. Rev. E, 74, 016103, doi:10.1103/PhysRevE.74.016103, 2006.

Papa, A. R. R. and Sosman, L. P.: Statistical properties of geomagnetic measurements as a potential forecast tool for strong perturbations, J. Atmos. Solar-Terr. Phys., 70, 1102-1109, doi:10.1016/j.jastp.2008.01.010, 2008.

Papa, A. R. R., Barreto, L. M., and Seixas, N. A. B.: Statistical study of magnetic disturbances at the Earth's surface, J. Atmos. Solar-Terr. Phys., 68, 930-936, doi:10.1016/j.jastp.2006.01.004, 2006.

Ramos, F. M., Bolzan, M. J. A., Sá, L. D. A., Rosa, R. R., and Rodrigues Neto, C.: Atmospheric turbulence within and above an Amazon Forest, Physica D-Nonlinear Phenomena, 193, 278291, doi:10.1016/j.physd.2004.01.026, 2004.
Richardson, I. G. and Cane, H. V.: Identification of interplanetary coronal mass ejections at $1 \mathrm{AU}$ using multiple solar wind plasma composition anomalies, J. Geophys. Res., 109, A09104, doi:10.1029/2004JA010598, 2004.

Rodrigues Neto, C., Zanandrea, A., Ramos, F. M., Rosa, R. R., Bolzan, M. J. A., and Sá, L. D. A.: Multiscale analysis from turbulent time series with wavelet transform, Physica A - Statistical Mechanics and its Applications, 295, 215-218, 2001.

Sahai, Y., Fagundes, P. R., Becker-Guedes, F., Bolzan, M. J. A., Abalde, J. R., Pillat, V. G., de Jesus, R., Lima, W. L. C., Crowley, G., Shiokawa, K., MacDougall, J. W., Lan, H. T., Igarashi, K., and Bittencourt, J. A.: Effects of the major geomagnetic storms of October 2003 on the equatorial and low-latitude F region in two longitudinal sectors, J. Geophys. Res., 110, A12S91, doi:10.1029/2004JA010999, 2005.

Telesca, L., Lapenna, V., Vallianatos, F., Makris, J., and Saltas, V.: Multifractal features in short-term time dynamics of ULF geomagnetic field measured in Crete, Greece, Chaos, Solitons and Fractals, 21, 273-282, doi:10.1016/j.chaos.2003.10.020, 2004.

Vörös, Z:: On multifractality of high-latitude geomagnetic fluctuations, Ann. Geophys., 18, 1273-1282, doi:10.1007/s00585-0001273-6, 2000.

Vörös, Z., Jankovičová, D., and Kovács, P.: Scaling and singularity characteristics of solar wind and magnetospheric fluctuations, Nonlin. Processes Geophys., 9, 149-162, doi:10.5194/npg9-149-2002, 2002.

Wanliss, J. A., Anh, V. V., Yu, Z.-G., and Watson, S.: Multifractal modeling of magnetic storms via symbolic dynamics analysis, J. Geophys. Res., 110, A08214, doi:10.1029/2004JA010996, 2005.

Weygand, J. M., Kivelson, M. G., Khurana, K. K., Schwarzl, H. K., Thompson, S. M., McPherron, R. L., Balogh, A., Kistler, L. M., Goldstein, M. L., Borovsky, J., and Roberts D. A.: Plasma sheet turbulence observed by CLUSTER II, J. Geophys. Res., 110, A01205, doi:10.1029/2004JA010581, 2005. 